\title{
Solar Neutron and Gamma-ray Detector for a 3U CubeSat
}

\author{
Kazutaka Yamaoka*, Hiroyasu Tajima, Kikuko Miyata, Takaya Inamori, Yoshinori \\ Sasai, Hiroaki Kawahara, Ji Hyun Park, Kazuhiro Nakazawa, Satoshi Masuda, Koji \\ Matsushita, Kazuya Itoh, Daiki Nobashi
}

Nagoya University, Nagoya, Japan

E-mail: vamaokadisee.nagoya-u.ac.jp

\section{Hiromitsu Takahashi}

Hiroshima University, Higashi-Hiroshima, Japan

\section{Kyoko Watanabe}

National Defense Academy of Japan, Yokouska, Japan

\begin{abstract}
Solar neutron observations are very important on understanding of particle acceleration mechanism in the Sun. However, previous ground-based observations with large area telescope $(\sim 10$ $\mathrm{m}^{2}$ )at high latitude are not sensitive to solar neutrons due to attenuation in the earth atmosphere and roughly 10 detection since its discovery in 1980. From space, the SEDA-AP instrument with much smaller area $\left(100 \mathrm{~cm}^{2}\right)$ onboard the International Space Station (ISS) monitored solar neutrons including charged particles, and successfully detected more than 30 detection since its launch in 2009. Unfortunately the SEDA-AP operation was stopped on March 2018. To overcome situation for no mission dedicated for solar neutrons, we have designed and developed a solar neutron and gamma-ray detector for a $3 U$ cubesat with a size of $30 \times 10 \times 10 \mathrm{~cm}$. Actually we launched the 50-kg class ChubuSat-2 satellite for solar neutron observations on February 2016, and have now been adjusting it to a 3U cubesat application. The solar neutron and gammaray detector consists of multi-layered plastic scintillator bars, and $\mathrm{GAGG}(\mathrm{Ce})$ scintillator array, and both of them are read out with silicon photo-multipliers (Si PMs). More than 600 signals from Si PMs are processed by ASICs. In this paper, we will describe details of the detector and performance of its breadboard model (BBM).
\end{abstract}

36th International Cosmic Ray Conference -ICRC2019-

July 24th - August 1st, 2019

Madison, WI, U.S.A.

\footnotetext{
* Speaker.
} 


\section{Introduction}

Solar flares are energetic explosion in the Sun, and found to be as a sudden increase of X-rays. Particle acceleration is frequently observed in association with strong solar flares, and associated magnetic storms sometimes affect human life. It is believed that magnetic reconnection is very important process in solar surfaces, [四] but no one knows details of how, when and where particles accelerated. Several scenario such as shock acceleration in the reconnection region and stochastic acceleration in the turbulance has been proposed. Previous observations of solar flares have been mainly observed with electro-magnetic waves from radio to $\mathrm{X}$ - and gamma-rays, but they are basically dominated by contribution of electrons. Charged particles such as protons and electrons are strongly affected by magnetic field in solar surface and interstellar medium, and lost original information about its energy and incident direction. Solar neutrons with an energy of $100 \mathrm{MeV}$ can reach the Earth within the time which is comparable to the life time ( $\sim 890 \mathrm{sec})$, and not affected by the magnetic field, hence, they can be only probe to understand nucleon acceleration mechanisms. Production of solar neutrons via interactions of nucleons with solar atmosphere were first predicted in 1950's, [[] but first detection of solar neutrons was made by NaI and CsI scintillators onboard Solar Maximum Mission (SMM) on June 21, 1980. [3] Solar neutron observations have been made by ground-based telescopes located at high altitude of several $1000 \mathrm{~km}$ to avoid attenuation by the Earth atmosphere. [ [ [ [ ] World-wide network of solar neutron telescopes with an area of more than $1 \mathrm{~m}^{2}$ have been constructed, but the sensitivity is not so good. Since August 2009, Space Environment Data Acquisition equipment - Attached Payload (SEDA-AP) FIBer detector on the International Space Station (ISS) has observed solar neutrons in the range of 30-120 MeV from space, [ $[6,0]$ and ended operation on April, 2018. So far there are in total about 40 detection (about 12 detection from the ground [ $[8]$ and about 30 detection from space), and no space-based mission is planned in the near future.

To overcome very severe situation, we have been developping micro/nano-satellite dedicated for solar neutron observation. The first opportunity was $50-\mathrm{kg}$ class microsatellite ChubuSat-2. A solar neutron detector was built at facilities of Nagoya University, and was launched as a piggyback satellite with the X-ray astronomical satellite ASTRO-H on February 17, 2016. [Q] However due to failure in the bus system, the detector was not turned on. Hence we are now trying to recover the mission for a $3 \mathrm{U}$ CubeSat in the near future. Recently we name this project as SONGS (SOlar Neutron and Gamma-ray Spectroscopy) Mission. In this paper, we describe details of the solar neutron detector for a small satellite. We introduce concept and design of the solar neutron detector in Section 2, realized detector for ChubuSat-2 in Section 3, and several improvements for a 3U CubeSat application in Section 4.

\section{Concept of our detector}

\subsection{Design and Detection Principle}

Our solar neutron detector was originally designed based on the SEDA-AP FIB. The FIB is multi-layered, thin plastic scintillator bars $(32 \times 16$ layers, $3 \mathrm{~mm} \times 6 \mathrm{~mm} \times 96 \mathrm{~mm}$ for each) readout by multi-anode photo-multiplier tubes (MA-PMTs). The bars are surrounded with anti-coincidence detectors to reject charged particles. To adopt the FIB to small satellite applications, we utilize 
novel semiconductor photo-device, silicon photomultiplier (Si PM), instead of MA-PMT. The Si PM has high gain $\left(\sim 10^{6}\right)$ and high quantum efficiency operated with lower voltage $(<100 \mathrm{~V})$ than PMT. We further add a function of gamma-ray detection by placing GAGG $\left(\mathrm{Gd}_{3} \mathrm{Al}_{2} \mathrm{Ga}_{3} \mathrm{O}_{12}\right.$; $\mathrm{Ce}$ ) inorganic scintillator array at the bottom of the detector. The schematic view of our detector is shown in Figure 1. Fast neutrons react in elastic scattering with hydrogen atoms in plastic scintillator bars, and recoiled proton loses its energy via ionization loss. Using scattering angle $\theta$ and total energy loss $E_{\mathrm{p}}$, we can estimate incident neutron energy as $E_{\mathrm{n}}=E_{\mathrm{p}} / \cos ^{2} \theta$ in nonrelativistic case. Gamma-rays are detected via Compton scattering process. Compton-scattered photon in the plastic scintillator bar is photo-absorbed in the inorganic scintillator array. Using positions and energies at two reaction points, we can estimate incident gamma-ray energy and constrain the direction in principle of Compton camera techniques. We can also detect gamma-ray photons directly with the inorganic scintillaion array after passing through the plastic scintillator bars (photo-absorption mode).

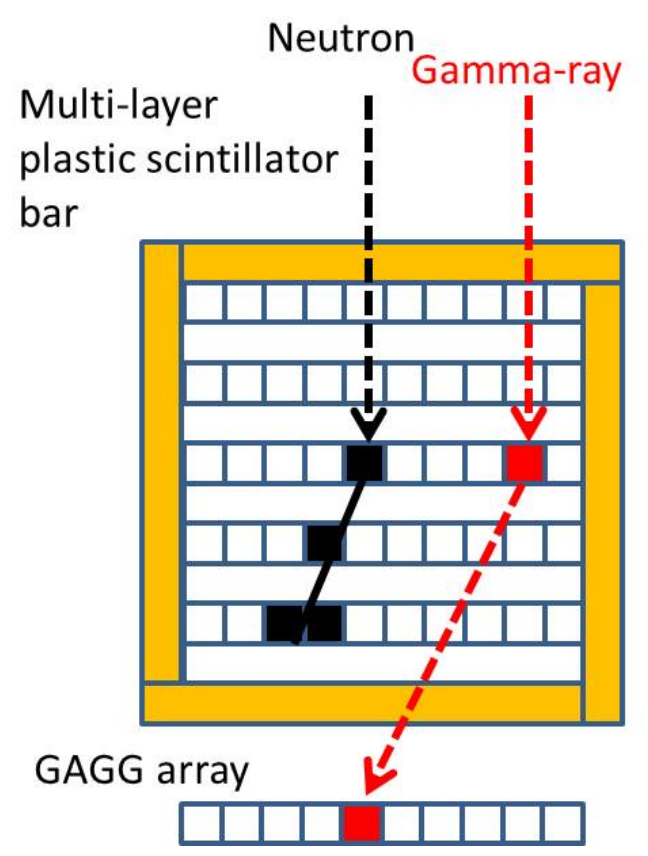

Figure 1: Schematic view of the solar neutron and gamma-ray detector. The detector consists of multilayered plastic scintillator bars, GAGG(Ce) inorganic scintillator array, and surrounding anti-coincidence shield to reject charged particles (shown by orange). Our detector is sensitive to both neutrons and gammarays. Neutrons are detected via elastic scattering with atoms in the plastic scntillator bars, while gamma-rays are detected via Compton scattering.

\subsection{Merits in Small Satellte Observations}

The SEDA-AP FIB observation was affected by secondary neutron background produced on the ISS, although the FIB was extended from the SEDA-AP experiment and located apart from the ISS structure after the launch. Since secondary neutron flux is roughly proportional to amount of surrounding materials, micro/nano-satellites $(<100 \mathrm{~kg})$ are expected to have much lower background than ISS with huge mass (420 ton). Neutron fluxes are significantly attenuated by the Earth 
atmosphere. The attenuation factor above $100 \mathrm{MeV}$ is $1 / 100$ to $1 / 1000$ depending on the altitude, and lower energy than $100 \mathrm{MeV}$ is not accessible from the ground. This means that the neutron flux on ground-based telescope with a large area of $1-10 \mathrm{~m}^{2}$ is comparable to that measured by space detector with a smaller area of $100 \mathrm{~cm}^{2}$. Hence, even smaller detector in space can achieve higher sensitivities than ground-based detectors. Actually the SEDA-AP has detected solar neutron events from M-class flares as wells as intense X-class flares. Furthermore, if the pointing direction is always toward the Sun, detector on the satellite can monitor the Sun with uniform sensitivity continuously.

Finally we emphasize that the scientific motivation and original design concept were proposed by graduate students who belong to leading graduate school program "Leadership Development Program for Space Exploration and Research" selected by Japan Society for the Promotion of Science (JSPS). [U]] This program began from 2013 to cultivate international leaders in spacerelated fields after getting Ph.D degrees. A small satellite can be very useful tool in terms of project management, actual design and development for students in the educational program.

\section{ChubuSat-2 Detector}

The ChubuSat is a series of 50-kg microsatellite developed by a collaboration among Nagoya University, Daido University, and small/medium-sized aerospace industry companies, so-called MASTT (Monozukui Aerospace Support Technology Team) around the Chubu region in Japan. The ChubuSat-2 is the 2nd ChubuSat following ChubuSat-1 launched in 2014. This mission is dedicated for monitoring radiation in space to support ASTRO-H celestial observations, and also observing solar neutrons. It has been accepted by JAXA as one of the four piggy-back satellites of ASTRO-H in summer 2014, and has been launched by H-IIA rocket No.30 from JAXA Tanegashima Space Center (TNSC) on February 17, 2016. The satellite was successfully deployed from the rocket, and communication between the satellite and ground station was well established via amateur radio band (VHF and UHF). But during initial checking phase within a week after the launch, we had trouble in communication between Power Supply Unit (PSU) and On- Board Computer (OBC) and the mission instrument had not been turned on after that. The solar neutron detector, called as a radiation detector (RD), and the satellite were built in a collaboration with Clear Pulse Co., Ltd. for only 1.5 years at facilities of Nagoya University. The realized detector is shown in Figure 2. Sensors and electronics are packaged in one aluminum box with a size of $15 \mathrm{~cm} \times 17 \mathrm{~cm} \times 18.5 \mathrm{~cm}$. The plastic scintillator part is 10x10 layers, and each bar size is 10 $\mathrm{mm} \times 10 \mathrm{~mm} \times 100 \mathrm{~mm}$. For readout of sensors, a Multi-Pixel Photon Counter (MPPC produced by Hamamatsu Photonics K.K.) with $6 \times 6 \mathrm{~mm}^{2}$ area is optically coupled to both sides of each bar to have a position sensitivity in the bar direction. MPPC current signals are sent to the electronics boards, and processed by charge-sensitive amplifiers, shaping amplifiers, lower discriminators, and 14-bit analog-to-digital converters (ADCs). The electronics board contains DC/DC converters to provide bias voltages $+55 \mathrm{~V}$ for MPPC and digital-to-analog converters (DACs) to adjust the gain of individual MPPCs. The GAGG scintillator part is $10 \times 10$ array with $10 \mathrm{~mm}$ cubic for each. Its signal is processed by the same MPPCs and electronics boards as used in plastic scintillator bars. In this configuration, there are in total 312 signals consisting of 200 for plastic scintillator bars, 100 for GAGG array, and 12 for anti-coincidence detectors. When some signals are found in the 
trigger, all the digitized pulse height information are collected to the Digital Processor (DP) board and the data are sent to the OBC. The specification of the ChubuSat-2 RD is summarized in Table 1 .
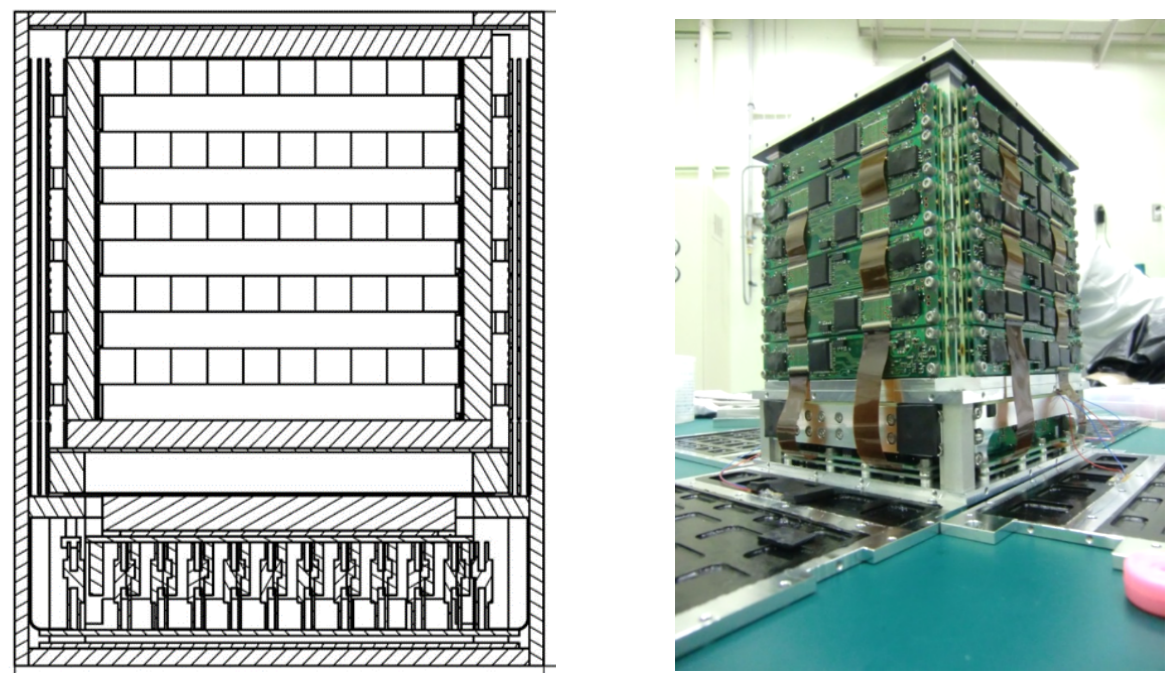

Figure 2: (Left) Structure design of the ChubuSat-2 radiation detector (RD). (Right) Picture of the ChubuSat-2 flight model detector fabricated in 2015. Outer alminium housing is removed from the detector. Sensors (scintillators and MPPCs) located inside the detector are surrounded with signal processing boards located at four sides and bottom.

Table 1: Specification of the ChubuSat-2 Radiation Detector.

\begin{tabular}{ll}
\hline \hline Items & Values \\
\hline Detector & 100 plastic scintillator bars + 10×10 GAGG (Ce) \\
& scintillator array readout with Multi-Pixel Photon Counter (MPPC) \\
Geometrical Area & $100 \mathrm{~cm}^{2}$ \\
Energy Range & $30-120 \mathrm{MeV}$ (neutrons), 200-1000 keV (gamma-rays) \\
Detection Efficiency & $1-2 \%$ for both neutrons and gamma-rays \\
Time resolution & $1 \mathrm{msec}$ \\
Nominal Power Consumption & $12 \mathrm{~W}$ \\
Data size to be downlinked & $\sim 10$ Mbyte per day by the S-band communication system \\
\hline
\end{tabular}

\section{3U CubeSat Detector}

In order to recover the solar neutron observation mission as promptly as possible, we have started a 3U CubeSat project since 2018. A CubeSat is generally cheaper and easier to build in shorter term than $50-\mathrm{kg}$ satellite. The size of $3 \mathrm{U}$ is $10 \mathrm{~cm} \times 10 \mathrm{~cm} \times 30 \mathrm{~cm}$ (the $1.2 \mathrm{U}$ size is roughly allocated for mission instruments and $1.8 \mathrm{U}$ size is for bus instruments). We have organized a new CubeSat team in collaboration with researchers at department of aerospace engineering who have many experience in CubeSat development. However, there are more severe resource constraints such as size, weight, and power in CubeSat instruments. The constraints are summarized in Table 
2 in comparison with characteristics of SEDA-AP FIB and ChubuSat-2 RD. The main change of the CubeSat sensor from the ChubuSat-2 RD is 1) to change the size of detector and 2) to reduce the power by a use of an Application Specification Integrated Circuit (ASIC). To adjust our detector to the $1.2 \mathrm{U}$ size $(10 \mathrm{~cm} \times 10 \mathrm{~cm} \times 12 \mathrm{~cm})$, we have designed the plastic scintillator part to be $64 \mathrm{~mm}$ cubic, but with finer structures than that of the ChubuSat-2 RD. We have studied energy resolution and detection efficiency using GEANT4 Monte-Carlo simulation (see Figure 3). [W]] We selected events with the criterion that recoiled proton tracks are identified at least 2 layers. The detection efficiency is roughly $1 \%$ which is worse by a factor of 2 than that of SEDA-AP. Since the detection number of solar neutrons is basically determined by a detector volume, it will be reduced to roughly $20 \%$ of that of SEDA-AP FIB, but still detectable by the CubeSat. We will also seek for a possibility to use GAGG in neutron detection which has Gadolinium sensitive to thermal neutron. On the other hand, the energy resolution of incident neutrons is better than that of SEDA-AP FIB because it is determined by geometrical size of plastic scintillator bar and we used a bit finer bar ( 4 $\mathrm{mm} \times 4 \mathrm{~mm} \times 64 \mathrm{~mm})$ than FIB $(3 \mathrm{~mm} \times 6 \mathrm{~mm} \times 96 \mathrm{~mm})$.

Table 2: Comparison among SEDA-AP FIB, ChubuSat-2 RD, and the CubeSat sensor.

\begin{tabular}{cccc}
\hline \hline Items & SEDA-AP FIB & ChubuSat-2 RD & CubeSat Sensor \\
\hline Satellite Size & (ISS) & $50 \mathrm{~cm}$ cubic & $10 \times 10 \times 30 \mathrm{~cm}(3 \mathrm{U})$ \\
Detector Size & $53.2 \times 53.2 \times 17.1 \mathrm{~cm}$ & $15 \times 17 \times 18.5 \mathrm{~cm}$ & $10 \times 10 \times 12 \mathrm{~cm}$ \\
Weight $(\mathrm{kg})$ & 12.7 & 6.2 & 2 \\
Power $(\mathrm{W})$ & 27.4 & 12 & 3 \\
Plastic Scinti. & $96 \mathrm{~mm}$ cubic $(512 \mathrm{pcs})$ & $100 \mathrm{~mm}$ cubic $(100 \mathrm{pcs})$ & $64 \mathrm{~mm}$ cubic $(256 \mathrm{pcs})$ \\
& $3 \times 6 \times 96$ mm for each & $10 \times 10 \times 100 \mathrm{~mm}$ for each & $4 \times 4 \times 64 \mathrm{~mm}$ for each \\
GAGG(Ce) & No instllation & $10 \times 10$ array & $12 \times 12$ array \\
& & 10 mm cubic for each & 6 mm cubic for each \\
Photosensor & Multi-Anode PMT & MPPC $6 \times 6 \mathrm{~mm}^{2}$ & MPPC $3 \times 3 \mathrm{~mm}^{2}(\mathrm{plastic})$ \\
& & for both plastic & $4 \times 4$ array with $6 \times 6 \mathrm{~mm}^{2}$ \\
& & and GAGG & each $(\mathrm{GAGG})$ \\
\hline
\end{tabular}

We also need to reduce power consumption from $12 \mathrm{~W}$ for ChubuSat- 2 to only $3 \mathrm{~W}$ for CubeSat significantly. The main reason for large power consumption of $12 \mathrm{~W}$ is that we used discrete electronic components such as operational amplifiers, comparators, ADCs, and so on. For the $3 \mathrm{U}$ CubeSat, we will use ASICs for readout of Si PMs provided by Integrated Detector Electronics AS (IDEAS) in Norway. The ASIC IDE3380 can process 16-channel MPPC signals with very low power (less than $2 \mathrm{~mW}$ per channel) and contain trigger generation circuits, amplifiers with gain adjustment, and ADC. Due to finer structure of the sensors than the ChubuSat-2 RD, we need to process in total 668 signals from sensors (512 from plastic scintillator bars, 144 from GAGG array, and 12 from anti-coincidence scintillators). The total power consumption can be satisfied within 3 W even for such number of signals. We have tested GAGG (Ce) $4 \times 4$ array readout with the ASIC evaluation board by irradiating gamma-ray radio-active isotopes. The GAGG (Ce) $4 \times 4$ array with $6 \mathrm{~mm}$ cubic for each is provided by Furukawa Electric Co., Ltd., and attached to the MPPC $4 \times 4$ array S13361-6050AE-04 produced by Hamamatsu Photonics K.K. We successfully read out all the signals from GAGG $4 \times 4$ array (Figure 4) and achieved excellent FWHM (Full-Width Half 

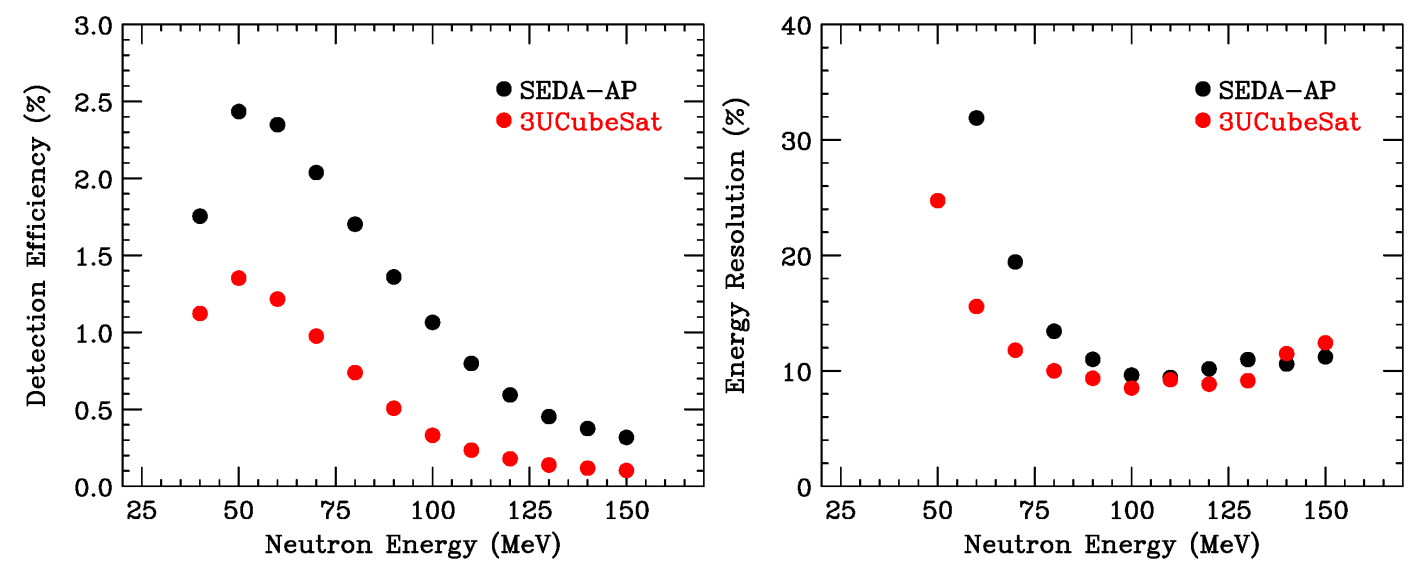

Figure 3: (Left) Detection efficiency of the CubeSat Neutron Sensor as a function of energy (shown by red) in comparsion with SEDA-AP FIB (in black). (Right) Energy resolution of neutrons as a function of energy.

Maximum) energy resolution of $\sim 7 \%$ at $662 \mathrm{keV}$. This result was obtained by an evaluation circuit, and we have also designed new original ASIC board combined with Field Programmable Gate Arrays (FPGAs) for digital signal processing. The size of this electric circuit board is adjusted to the CubeSat size, and the test is under way. As a schedule, the Bread Board Model (BBM) and Flight Model (FM) will be constructed in 2019 and 2021 respectively. The BBM consists of plastic scintillator bars with 4 layers and GAGG $4 \times 4$ array readout by the new ASIC board.
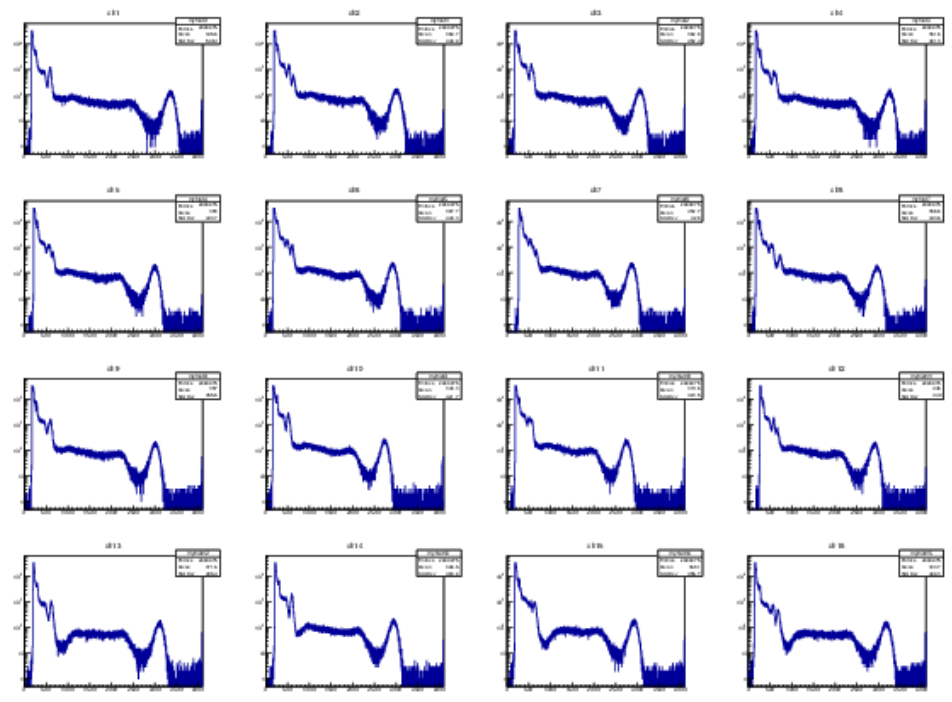

Figure 4: ${ }^{137} \mathrm{Cs}$ spectra are taken from this measurement system. The $662 \mathrm{keV}$ peak is clearly visible in all the 16-channel spectra, and the FWHM energy resolution is around $7 \%$ at $662 \mathrm{keV}$.

\section{Conclusion}

In order to realize high sensitive solar neutron observations, we have designed novel solar 
neutron and gamma-ray detector with very compact size, low power consumption, and light weight using the Silicon photomultiplier for a small satellite. The detector design is scalable and applicable to any sizes e.g., $3 \mathrm{U}$ CubeSat, 50-kg class satelite, and further large missions. A 3U CubeSat and its onboard detector are now under development, and we are currently aiming to launch the $3 \mathrm{U}$ CubeSat SONGS in 2022.

\section{Acknowledgments}

This research has been financially supported by Toyoaki Scholarship Foundation and JSPS Grant-in-Aid for Scientific Research (KAKENHI) Grant Number JP18H03700 (KY).

\section{References}

[1] Parker, E. N.: The Solar-Flare Phenomenon and the Theory of Reconnection and Annihiliation of MagneticFields, Astrophysical Journal Supplement, 8 (1963), pp. 177.

[2] Biermann, L., Haxel, O., \& SchlÃijter, A.: Neutral cosmic rays produced in the Sun, Zeitschrift fÃijNaturforschung, 6(1), (1951) pp. 47âĂŞ48.

[3] Chupp, E. L. Forrest, D. J., Ryan, J. M., et al.: A Direct Observation of Solar Neutrons Following the 0118 UTFlare on 1980 June 21, Astrophysical Journal, 263 (1982), pp. L95-L98.

[4] Matsubara, Y., Muraki, Y., Masuda, K, et al.: Observation of Solar Neutrons by the World-Wide Network ofSolar Neutron Detectors, Proceedings of the 26th International Cosmic Ray Conference, (1999), 42

[5] Sasai, Y., Nagai, Y., Itow, Y., et al.: Performance of the SciBar Cosmic Ray Telescope (SciCRT) toward theDetection of High-Energy Solar Neutrons in So-lar Cycle 24, Earth, Planet and Space, 66 (2014), pp. 130-137.

[6] Muraki, Y., Koga, K., Goka, T., Matsumoto, H., Obara, T., Okudaira, O., Shibata, S., Yamamoto, T.: Measurement by FIB on the ISS: Two Emissions of Solar Neutrons Detected?, Advances in Astronomy, (2012), article ID 379304.

[7] Koga, K., Muraki, Y., Masuda, S., Shibata, S., Matsumoto, H.; Kawano, H., Measurement of Solar Neutronson 05 March 2012, Using a Fiber-Type Neutron Monitor Onboard the Attached Payload to the ISS, SolaPhysics, 292(8) (2017), pp. 115-130.

[8] Yu, X. X., Lu, H., Chen, G. T., Li, X. O., Shi, J. K., Tan, C. M.: Detection of solar neutron events and theirtheoretical approach, New Astronomy, 39 (2015), pp. 25-35.

[9] Yamaoka, K., Babazaki, Y., Hayashi, Y., et al.: Solar Neutron and Gamma-ray Monitor on the ChubuSat-2Satellite, Transaction of the Japan Society for Aeronautical and Space Sciences (JSASS) AerospaceTechnology Japan 14(ists30), (2016), Pf_141-146.

[10] Leadership Development Program for Space Exploration and Research, Nagoya University Program for Leading Graduate Schools (LGS) web site: http://www.frontier.phys.nagoya-u.ac.jp/index-e.html (accessed January 1, 2012).

[11] Agostinelli, S., Allison, S., Amako, K., et al.: Geant4-a simulation toolki Nuclear Instruments aMethods in Physical Research Section A, 506 (3) (2003), pp. 250-303. 